\title{
Technical Note: Watershed strategy for oceanic mesoscale eddy splitting
}

\author{
Q. Y. Li and L. Sun
}

Key Laboratory of Atmospheric Composition and Optical Radiation, CAS, School of Earth and Space Sciences, University of Science and Technology of China, Hefei, 230026, China

Correspondence to: L. Sun (sunl@ustc.edu.cn)

Received: 18 May 2014 - Published in Ocean Sci. Discuss.: 30 June 2014

Revised: 12 January 2015 - Accepted: 9 March 2015 - Published: 20 March 2015

\begin{abstract}
To identify oceanic mononuclear mesoscale eddies, a threshold-free splitting method was developed based on the watershed. Because oceanic eddies are similar to plateaus and basins in the map of the sea level anomaly (SLA) data, the natural divisions of the basins are the watersheds between them. The splitting algorithm is based on identifying these watersheds by finding the path of steepest descent. Compared to previous splitting methods, the proposed splitting algorithm has some advantages. First, there are no artificial parameters. Second, the algorithm is robust; the splitting strategy is independent of the algorithm and procedure and automatically guarantees that the split mononuclear eddies are simply connected pixel sets. Third, the new method is very fast, and the time complexity is $O(N)$, where $N$ is the number of multinuclear eddy pixels; each pixel is scanned only once for splitting, regardless of how many extremes there are. Fourth, the algorithm is independent of parameters; the strategy can potentially be applied to any possible physical parameters (e.g. SLA, geostrophic potential vorticity, Okubo-Weiss parameter). Besides, the present strategy can also be applied to automatic identification of troughs and ridges from weather charts. Because this general method can be applied to a variety of eddy parameter fields, we denoted it the Universal Splitting Technology for Circulations (USTC) method.
\end{abstract}

\section{Introduction}

To investigate the dynamics and roles of oceanic eddies in the environment, these eddies must first be automatically identified and tracked, especially when they are close to each other. In general, the automated eddy detection algorithms are categorized into three types: (1) physical parameter-based algorithms, e.g. Okubo-Weiss (Isern-Fontanet et al., 2003; Chaigneau et al., 2008); (2) flow geometry-based algorithms (Fang and Morrow, 2003; Chaigneau et al., 2011; Petersen et al., 2013; Chelton et al., 2011; Xu et al., 2011; Wang et al., 2015); and (3) hybrid methods, which involve physical parameters and flow geometry characteristics (Nencioli et al., 2010, Xiu et al., 2010; Dong et al., 2011; Yi et al., 2014). However, each identification method poses a multinuclear eddy identification problem, e.g. multiple sea level anomaly (SLA) extremes (Chelton et al., 2011). This problem can occur when multiple eddies are physically close together. Note that such multiple eddies are very common in SLA data $(\mathrm{Li}$ et al., 2014; Wang et al., 2015).

A simple method to avoid the problem is to reduce the number of contours of the SLA until there is only one extreme in the contour (Chaigneau et al., 2011). Thus, only one extreme is located in the eddy, as shown in Fig. 1a. However, reducing this contour will lead to reductions in both the area and the amplitude of the eddy. The identified eddies are much smaller and weaker. For example, the amplitudes of the identified eddies were only approximately $2-3 \mathrm{~cm}$ (Chaigneau et al., 2008), whereas they could be in the range of 20 to $30 \mathrm{~cm}$ in other eddy identifications (Chelton et al., 2011; Xiu et al., 2010).

The best approach to solve the multinuclear eddy identification problem is by directly splitting multinuclear eddies, as shown in Fig. 1b. This splitting is not easily achieved. Chelton et al. (2011) attempted to split multinuclear eddies using various methods. However, their splitting process often resulted in some track problems, and it was finally abandoned. 


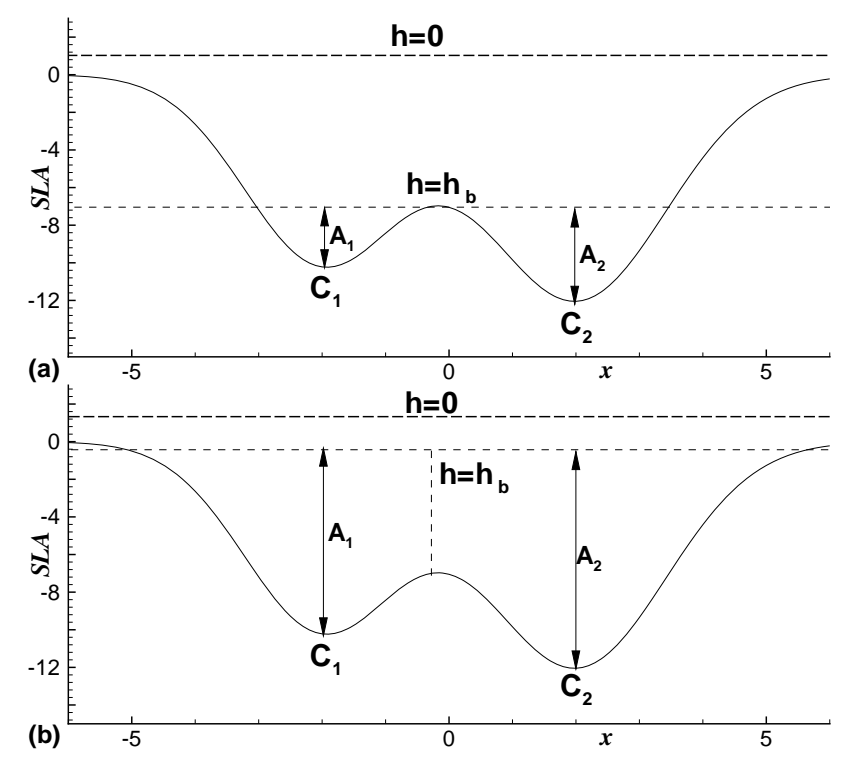

Figure 1. (a) Non-splitting mononuclear eddy identification. (b) Mononuclear eddy identification with splitting. Both the amplitude and the area are quite different in the two methods.

Subsequently, Yi et al. (2014) applied a hybrid detection approach by integrating the ideas of the Okubo-Weiss method and the SLA-based method. Li et al. (2014), following the approach proposed by Chelton et al. (2011), attempted to split multiple eddies according to SLA with two simple strategies and a threshold for strategy choice.

Note that Yi's hybrid method does not include any splitting strategy or method. As a result, Yi's hybrid method simply identifies the boundary of the multinuclear eddy using one parameter and identifies the centres of multinuclear eddies using another parameter but cannot actually split multinuclear eddies into single ones. Li's method, which includes the splitting method, requires an additional threshold. In addition, these splitting methods have difficulty in identifying very close multinuclear eddies.

The goal of this study was to establish a splitting strategy that could separate multinuclear eddies into mononuclear eddies. The idea is based on the fact that the values of eddy parameters (e.g. SLA) are similar to plateaus (anti-cyclonic eddies) and basins (cyclonic eddies) in a map and that the vortex is similar to a funnel like a black hole (Haller and Beron-Vera, 2013). The natural divisions of the basins are the watersheds between them. For basins, the "watershed" is a ridge between them, while it is a valley for plateaus.

In this paper, we do not try to find the exact location of the watersheds, but rather we only use the property of watershed (ridge): a particle cannot roll across the ridge from one basin to another one. We use the valley (ridge) to split the anti-cyclonic (cyclonic) multi-nuclear eddy into mononuclear ones. To simplify the descriptions, we use only cyclonic eddies as examples. The anti-cyclonic eddies can be split in a similar way.

\section{Definition of a mononuclear eddy}

\subsection{Data}

The SLA data used in this study were from the MSLA (maps of sea level anomalies), a merged and gridded satellite product, which is produced and distributed by AVISO (archiving, validation, and interpretation of satellite oceanographic data at http://www.aviso.oceanobs.com/) and based on TOPEX/Poseidon, Jason 1, and the European remote sensing (ERS) satellites (i.e. ERS-1 and ERS-2 data) (Ducet et al., 2000). Currently, the products are available on a daily scale at a resolution of $0.25^{\circ} \times 0.25^{\circ}$ over the global ocean. The data were corrected for all geophysical errors.

\subsection{Mononuclear eddy identification}

To identify eddies, a physical definition of an eddy is required. In general, an eddy is considered a coherent structure characterized by water rotating around a common centre (Chelton et al., 2011; Faghmous et al., 2013) and a structure that retains all its initial mass as it propagates (Haller and Beron-Vera, 2013). Because this study focuses mainly on the splitting strategy, the choice of parameters is not of concern, and we simply use SLA as an example. The following mononuclear eddy definition is from previous studies ( $\mathrm{Li}$ et al., 2014). Each pixel has eight nearby neighbours. A point within the region is a local extremum if it has an SLA greater or less than all of its nearest neighbours. We also use such definition of extremum in our following studies, in which the extrema are identified by checking each pixel in the map and the eight pixels around them. An eddy is defined as a simply connected set of pixels that satisfies the following criteria:

1. Only one SLA extremum exists in the set.

2. The SLA values of the eddy are above (below) a given SLA threshold associated with data error, e.g. $3 \mathrm{~cm}$ for anti-cyclonic (e.g. $-3 \mathrm{~cm}$ for cyclonic) eddies.

3. The amplitude of the eddy is larger than the data error (e.g. $3 \mathrm{~cm}$ ).

Conditions (2) and (3) provide lower bounds for the eddy size and amplitude. Moreover, we increase the amplitude criterion from $1 \mathrm{~cm}$, as proposed by Chelton et al. (2011), to $3 \mathrm{~cm}$ because the SLA data error is approximately $3 \mathrm{~cm}$ (Ponte et al., 2007). The above criteria remove the constraints of eddy pixel number and distance between eddy pixels (e.g. Chelton et al., 2011). Therefore, they are simpler and more consistent.

The eddy is identified by the following procedures. First, we find a simply connected region with a given a threshold. Second, we check whether there is at least one extremum in 
the region. Then we check whether the region satisfies the eddy conditions (2) and (3). Finally, we check whether the eddy is multinuclear. As both conditions (2) and (3) allow the eddy to be multinuclear, we explicitly add condition (1) as a constraint. However, we need a splitting method to implement this.

\section{Eddy splitting method}

\subsection{Eddy splitting strategy}

In this study, an eddy is split based on the fact that the negative gradient vector of the SLA points toward the eddy centre of an ideal circular-shaped eddy (Li et al., 2014) and the fact that the vortex is similar to a funnel (Haller and Beron-Vera, 2013). Because oceanic cyclonic eddies are similar to basins in the map of the SLA data, the natural divisions of the basins are the watersheds between them.

Figure 2 illustrates this eddy splitting strategy. Figure 2a shows two individual but close eddies. The pixels between the two dashed lines are naturally divided by the watershed. As shown in Fig. 2b, the cross section of the eddy clearly shows that two closely located particles on the left and right sides of watershed slide along their ways to different eddy centres. The shape of SLA can provide sufficient information to split the multinuclear eddy into mononuclear ones.

To make the strategy more effective, we assume that all of the particles fall only along the path of steepest descent. This assumption ensures that the particle at each pixel has only one path to the eddy centre. As the path to the centre is mathematically well defined, it is obvious that such a path does not depend on the search method or procedure.

\subsection{Eddy splitting procedure}

A simple example of the splitting procedure for cyclonic multinuclear eddy is illustrated in Fig. 2c. The procedure for the anti-cyclonic one is similar but with a little bit of difference in Sect. 3.4. At first, the extremes with the definition in Sect. 2.2 are labelled as $C_{1}$ and $C_{2}$. Then, a path of steepest descent is found from the pixel $p_{a 1}$ to $C_{1}$. Finally, the pixels in the path are marked as $\mathrm{C} 1$ (i.e. the part of eddy $C_{1}$ ). Similarly, the pixel $p_{b 1}$ has the path of steepest descent to $p_{a 3}$ (which is already marked as $C_{1}$ ); thus it is also marked as $C_{1}$. We describe the above procedure as in the following algorithm. For any cyclonic multinuclear eddy, the following steps are taken:

1. Label the extremes as cyclonic eddies of $C_{1}, C_{2}, C_{3}$, etc.

2. Mark the pixels in the multinuclear eddy as $1,2,3, \ldots$, $n$.

3. Let the index $i=1$.
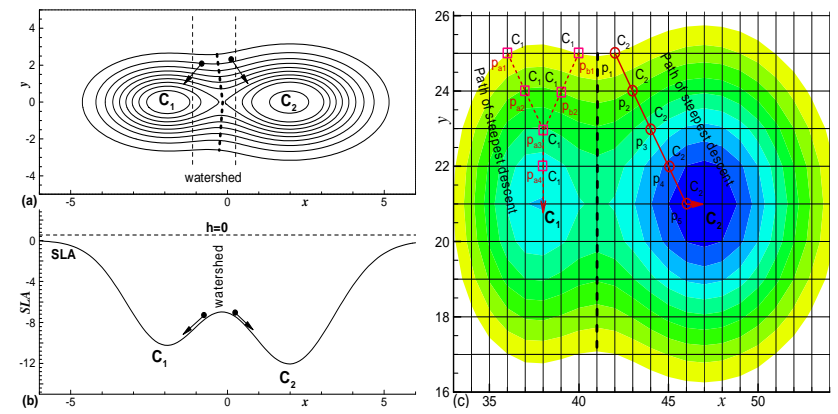

Figure 2. (a) The watershed as the natural division of eddies. (b) The particles on the watershed flow downward to the eddy centres. (c) Sketch map of the fast descent algorithm, where the dashed line indicates the watershed. The squires with arrows are paths to eddy $C_{1}$, while the circles with arrows are paths to eddy $C_{2}$.

4. Take the $i$ th pixel from the list.

5. Is it marked as part of any eddy? If yes, go to (8). If no, go to (6).

6. Find the path and eddy label " $\mathrm{Cx}$ " for the $i$ th pixel using the fast descent method.

7. Mark all of the pixels in the path as cyclonic eddy " $\mathrm{Cx}$ ".

8. Let the index $i=i+1$; if $i>n$, go to (9); otherwise go to $(5)$.

9. Stop.

The splitting procedure has two obvious advantages. First, this procedure automatically guarantees that the split mononuclear eddies are simply connected pixel sets because all the pixels in the eddy are connected to the central extremum. In contrast, the previous splitting methods cannot guarantee this connected nature, and another further procedure is needed to delete the unconnected parts ( $\mathrm{Li}$ et al., 2014).

Second, the algorithm is linear and very fast. Each pixel is scanned only once; thus, the time complexity is $O(N)$, where $N$ is the number of multinuclear eddy pixels. However, the split method is not completely finished. In step (6), we require a procedure to return the path from any pixel to eddy "Cx".

\subsection{Path of steepest descent}

In the splitting procedure, we need to find the path of steepest descent. Noting that each pixel is surrounded by eight discrete neighbours, the paths are only the connections of the nearest pixels with approximation, when the particles roll straight downhill (in continuous field). A simple example of such a path is illustrated in Fig. 2c. The arrows indicate the path of steepest descents from pixel $p_{1}$ to the eddy centre $C_{2}$ . In the algorithm, the path of steepest descent from pixel " $i$ " can be obtained through the following steps: 


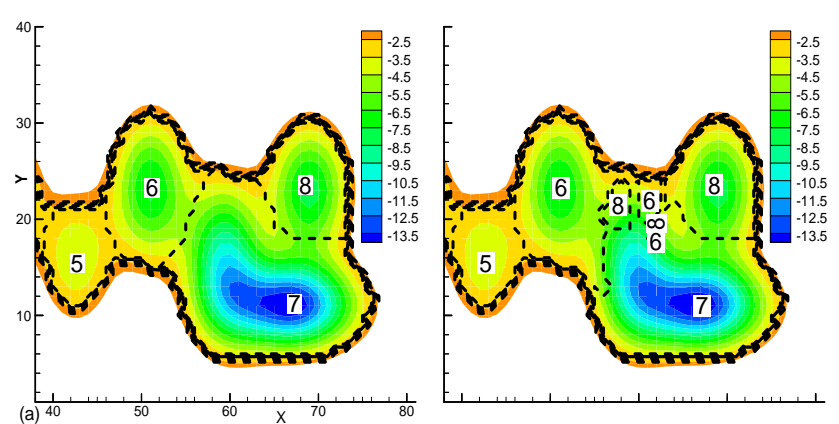

Figure 3. (a) Example of division of a multi-nuclear eddy by present algorithm, where the colour contours represent the SLA, and the numbers identify each eddy. (b) The same example as in (a) but by previous splitting strategies. The eddy boundaries are more zigzag in nature at the vicinity of eddies 6,7 and 8 than those in (a). Besides, both eddies 6 and 8 have disconnected areas after splitting.

\section{Let $m=1$.}

2. Take pixel " $i$ " as the $m$ th element of the path.

3. Find the pixel " $j$ " with the lowest value amongst " $i$ " and the surrounding eight pixels.

4. Check whether " $j$ " is already marked as " $\mathrm{Cx}$ ". If yes, go to (6). If no, go to (5).

5. Let $m=m+1$ and $i=j$, go to (2).

6. Return along the path of $m$ pixels and label those pixels as parts of eddy " $\mathrm{Cx}$ ".

\section{Stop.}

This procedure returns the path of steepest descent of a pixel to the eddy extremum. If a node of the path (e.g. $p_{a 3}$ ) has already been marked as part of an eddy (e.g. $C_{1}$ ), it will return the result immediately. As a result, this procedure is very efficient and fast. In step (3), the pixel with the lowest value is well defined. Therefore, the path of steepest descent to the eddy extremum is also well defined. There is only one path of steepest descent for any pixel, and this path is independent of the search procedure. As a result, the procedure is independent of the scan order and is thus robust.

\subsection{The example}

We apply this method to some examples. Figure 3a shows four cyclonic eddies that are difficult to split because they are very close to each other. Li et al. (2014) suggested reidentifying a multinuclear eddy if too many extremes exist $(n>3)$. The present algorithm can simply split the multinuclear eddy into individual ones, using the watersheds between each eddy as the eddy boundaries. We also used Li's method to split the multinuclear eddy, and the result is shown in Fig. 3b. Compared to the present algorithm, the previous
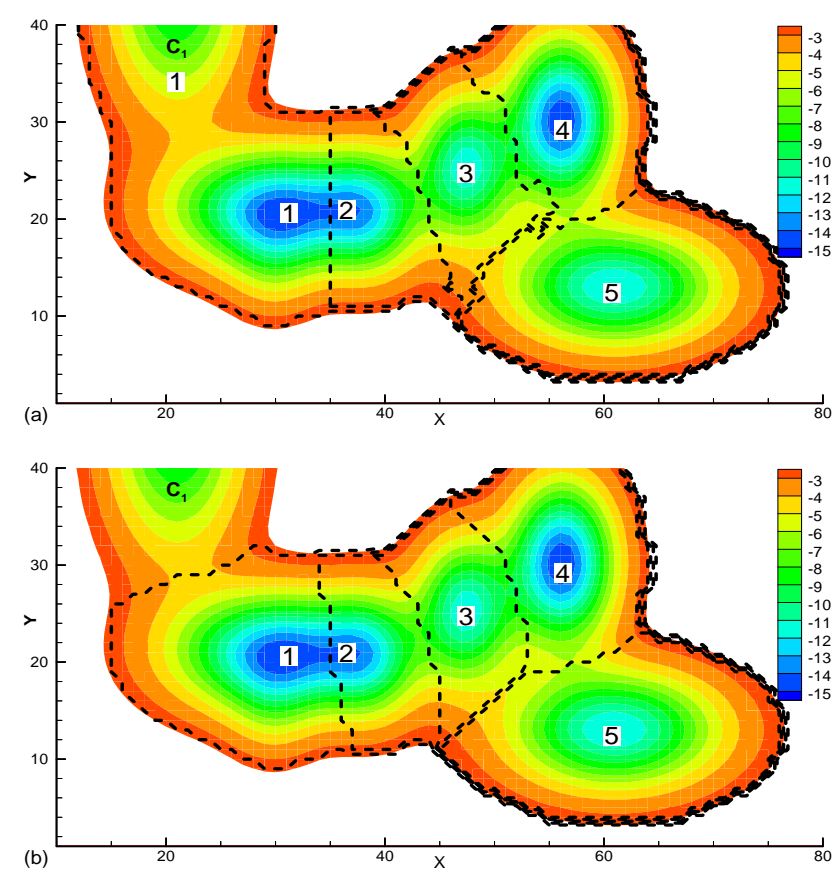

Figure 4. (a) Example of eddy splitting in simply connected region, where the colour contours represent the SLA, and the numbers identify each eddy. Part of an eddy $C_{1}$ is located at $(10<x<30$, $30<y<40)$ in this region. It was recognized as part of eddy 1 according to previous methods. (b) Same example as in (a) but by present splitting strategy. The new algorithm automatically eliminates eddy $C_{1}$ from the present region. The eddy boundaries are smoother in nature than those in (a).

method can also split the multinuclear eddy into four individual ones, but the result is quite different from that obtained with the proposed algorithm except for eddy 5 . First, eddies 6 and 8 have disconnected areas, and eddy 7 exhibits multiple connections after the splitting procedure; as a result, an additional procedure is required to eliminate this issue. Second, the eddy boundaries are more zigzag in appearance than those shown in Fig. 3a. The twisted eddy shape will introduce some difficulties in further applications. For example, the eddy composition must initially find similarly shaped eddies.

However, this new method can also avoid another problem in many SLA-based identification methods. As shown in Fig. 4a, the colour contours show a simply connected region above a critical value. Part of an eddy $C_{1}$ is located at $[10<x<30,30<y<40]$ in this region. It is recognized as part of eddy 1 according to previous methods. However, the present method can automatically recognize it as part of another eddy (Fig. $4 \mathrm{~b}$ ) because there is a watershed between eddy $C_{1}$ and eddy 1.

When the eddies are anti-cyclonic like plateaus, the above method cannot be directly used. One may transform the SLA values into negative ones by multiplying by -1 . This data transform is only for eddy splitting. The above method is 
valid for these modified data. Alternatively, we can also use the fast ascend method to split the anti-cyclonic eddies by noting that the extremes are local maximal, and that the watersheds are valleys now.

In general, the splitting strategy should meet the following requirements. First, the strategy should be threshold-free. Any artificial threshold might be unphysical and controversial. Second, the strategy should be robust: the splitting strategy should be independent of the numbers of extremes and independent of the algorithm and procedure. Third, the strategy should be independent of the parameter(s) usable. Because there are many eddy parameters (e.g. SLA, geostrophic potential vorticity, Okubo-Weiss parameter), the best parameter for the physical definition of an eddy remains unknown. The present algorithm satisfies all of these requirements.

\section{Conclusions}

In this study, a watershed splitting strategy was used for mononuclear eddy identification. The splitting strategy has the following advantages. First, the strategy is thresholdfree. No artificial threshold was required in the proposed procedure. Second, the strategy is robust and independent of the algorithm and procedure used. Third, the strategy is very fast, regardless of how many extremes there are. Fourth, the strategy is independent of the parameter used (e.g. SLA, geostrophic potential vorticity, Okubo-Weiss parameter). In addition, the present strategy can be applied to automatic identification of troughs and ridges from weather charts. Due to the potential general applications of eddy splitting, we denoted it the Universal Splitting Technology for Circulations (USTC) method.

Acknowledgements. We thank D. G. Bowers and another referee for comments and suggestions. This work was supported by the National Basic Research Program of China (no. 2013CB430303) and the National Foundation of Natural Science (no. 41376017). We thank AVISO for providing the SLA data.

Edited by: O. Zielinski

\section{References}

Chaigneau, A., Gizolme, A., and Grados, C.: Mesoscale eddies off Peru in altimeter records: identification algorithms and eddy spatio-temporal patterns, Progr. Oceanogr, 79, 106-119, 2008.

Chaigneau, A., Le Texier, M., Eldin, G., Grados, C., and Pizarro, O.: Vertical structure of mesoscale eddies in the eastern South Pacific Ocean: A composite analysis from altimetry and Argo profiling floats, J. Geophys. Res.-Oceans, 116, C11025, doi:10.1029/2011JC007134, 2011.
Chelton, D. B., Schlax, M. G., Samelson, R. M., and de Szoeke, R. A.: Global observations of large oceanic eddies, Geophys. Res. Lett., 34, L15606, doi:10.1029/2007GL030812, 2007.

Chelton, D. B, Schlax, M. G., and Samelson, R. M.: Global observations of nonlinear mesoscale eddies, Progr. Oceanogr., 91, 167-216, 2011.

Dong, C., Nencioli, F., Liu, Y., and McWilliams, J.: An automated approach to detect oceanic eddies from satellite remotely sensed sea surface temperature dat, IEEE Geosci. Remote S., 99, 1-5, 2011.

Ducet, N., Le Traon, P. Y., and Reverdin, G.: Global high resolution mapping of ocean circulation from TOPEX/Poseidon and ERS-1 and-2, J. Geophys. Res., 105, 19477-19478, doi:10.1029/2000JC900063, 2000.

Faghmous, J. H., Uluyol, M., Styles, L., Le, M., Mithal, V., Boriah, S., and Kumar, V.: Multiple Hypothesis Object Tracking For Unsupervised Self-Learning: An Ocean Eddy Tracking Application, In Proceedings of the Twenty-Seventh AAAI Conference on Artificial Intelligence, 1277-1283, 2013.

Fang, F. and Morrow, R.: Evolution, movement and decay of warmcore Leeuwin Current eddies, Deep-Sea Res. II, 50, 2245-2261, 2003.

Haller, G. and Beron-Vera, F. J.: Coherent Lagrangian vortices: The black holes of turbulence, J. Fluid Mech., 731, R4, doi:10.1017/jfm.2013.391, 2013.

Isern-Fontanet, J., Garcia-Ladona, E., and Font, J.: Identification of marine eddies from altimetric maps, J. Atmos. Ocean. Tech., 20, 772-778, 2003.

Li, Q. Y., Sun, L., Liu, S. S., Xian, T., and Yan, Y. F.: A new mononuclear eddy identification method with simple splitting strategies, Rem. Sens. Lett., 5, 65-72, 2014.

Nencioli, F., Dong, C., Dickey, T., Washburn, L., and McWilliams, J. C.: A vector geometry-based eddy detection algorithm and its application to a high-resolution numerical model product and high-frequency radar surface velocities in the Southern California Bight, J. Atmos. Ocean. Tech., 27, 564-579, 2010.

Petersen, M. R., Williams, S. J., Maltrud, M. E., Hecht, M. W., and Hamann, B.: A threedimensional eddy census of a highresolution global ocean simulation, J. Geophys. Res.-Oceans, 118, 17591774, 2013.

Ponte, R. M., Wunsch, C., and Stammer, D.: Spatial Mapping of Time-Variable Errors in Jason-1 and TOPEX/Poseidon Sea Surface Height Measurements, J. Atmos. Ocean. Tech., 24, 10781085, 2007.

Wang, Z. F., Li, Q. Y., Sun, L., Li, S., Yang, Y. J., and Liu, S. S.: The most typical shape of oceanic mesoscale eddies from global satellite sea level observations. Front. Earth Sci., 9, doi:10.1007/s11707-014-0478-z, 2015.

Xiu, P., Chai, F., Shi, L., Xue, H., and Chao, Y.: A census of eddy activities in the South China Sea during 1993-2007, J. Geophys. Res.-Oceans, 115, C03012, doi:10.1029/2009JC005657, 2010.

Xu, C., Shang, X. D., and Huang, R. X.: Estimate of eddy energy generation/dissipation rate in the world ocean from altimetry data, Ocean Dynam., 61, 525-541, 2011.

Yi, J., Du, Y., He, Z., and Zhou, C.: Enhancing the accuracy of automatic eddy detection and the capability of recognizing the multicore structures from maps of sea level anomaly, Ocean Sci., 10, 39-48, doi:10.5194/os-10-39-2014, 2014. 